\title{
Optimización económica de los camiones asignados en la operación Dragalinas/Apron Feeder en una empresa minera de carbón en el departamento del Cesar
}

Sandra Molina', Mary Hernández ${ }^{2}$

\section{Resumen}

En el ámbito de la minería para que exista una óptima producción influyen muchos factores. En la operación Dragalina/Apron Feeder depende directamente del factor de camiones asignados, el cual tiene un gran impacto en la producción y costo de la operación. Mediante este artículo se busca determinar la saturación de la asignación de camiones, proporcionado por la tasa de producción óptima al menor costo por metro cúbico banco. Además, se determinará el número mínimo de camiones asignados, lo que produce un costo por metro cúbico banco por debajo del objetivo de corte de la empresa, desarrollando un modelo de costos a partir de los datos históricos y costos de producción en la mina, utilizando Excel para analizar el impacto que genera una buena asignación de camiones.

Palabras clave: asignación de camiones, óptima, saturación.

1'Ingeniera de minas de la Fundación Universitaria del Área Andina. Valledupar, Colombia. Correo: Smolina17@areandina.edu.co 2Estudiante de Ingeniería de Minas de la Fundación Universitaria del Área Andina. Valledupar, Colombia. Correo: mahernandez5010 estudiantes.area ndina.edu.co 


\section{Introducción}

En el ámbito de la minería para que exista una óptima producción depende de muchos factores, los cuales siempre están en una mejora continua, uno de estos son los ciclos de los camiones en el acarreo de roca y carbón. La producción de roca con el sistema de dragalinas/apron feeder es directamente dependiente del factor de camiones asignados. A medida que los tiempos de ciclo del camión se afectan, el número de asignación de camiones aumenta o disminuye. El objetivo de este proyecto es determinar la saturación de la asignación de camiones proporcionado por la tasa de producción óptima al menor costo por metro cúbico banco. Además, se determinará el número mínimo de camiones asignados, lo que produce un costo por metro cúbico banco por debajo del objetivo de corte de la empresa. Para lograr estos objetivos, se desarrollará un modelo de costos a partir de los datos históricos y costos de producción en la mina.

Las dragalinas son muy económicas y representan unos de los equipos más populares en cuanto a remoción de roca. Son comúnmente usadas en combinación con raspadores para reducir la cantidad de remanejo. Una dragalina es como la estructura de una grúa con un largo balde con capacidad de $100 \mathrm{~m}^{3}$, suspendida por cables de acero. El balde se arrastra a través de la roca y una vez se ha llenado se alza. La base de los pivotes de la dragalina y la roca se botan en otros lugares. La roca generalmente necesita ser volada antes de que la dragalina pueda ser usada. En el caso de DLTD no sucede así porque las dragalinas trabajan en material de aluvión de río. Las dragalinas son comúnmente usadas en minería a cielo abierto para remover la roca para exponer la traza de los mantos de carbón. Una dragalina es básicamente una grúa giratoria en la que el balde es suspendido entre los cables de izar y los cables de arrastre que estiran el balde hacia el eje de rotación. En un solo ciclo, la secuencia típica del movimiento es el siguiente: deje caer el balde, arrastre sobre la superficie a rellenar con roca suelta, izar el balde, llevar el boom de la draga a donde botan el material, en este caso en Drummond Ltd. en los alimentadores blindados, y hacer el giro de vuelta y llevar el balde a su posición de inicio.

Apron feeder es un alimentador blindado, es una máquina larga de material de acarreo para procesos por manufactura o un proceso industrial. 
Esta máquina ha sido reensamblada con cortas o pequeñas partes de bandas transportadoras, más conocidas como alimentadores de plato o banda blindada. Los alimentadores blindados permiten automáticamente el control del volumen del material, que es añadido al proceso, regulando la velocidad del material que se añade a los camiones. Los alimentadores blindados se pueden encontrar en operaciones mineras, fábricas y plantas de concreto.

DLTD apron feeder. La MMD diseñó alimentadores empleados por DLTD, generando gran impacto al cargue del material directamente al plato de los alimentadores, incluso cuando el alimentador estaba operando. Cuando el material era cargado en el alimentador los platos trituraban el material, absorbiendo las fuerzas de impacto inicial indefinidas y haciendo que estas se deformaran dentro de su límite elástico.

\section{Materiales y métodos}

La metodología se desarrolló inicialmente con un estudio de los antecedentes de los últimos años que han arrojado los datos históricos de la operación en las dragalinas/apron feeder, para la confinación de estos datos se realizarán estudios de los datos históricos de la productividad de estas mismas, visitas a campo, es decir, al área en la que operan y observar posibles afectaciones para la productividad. Para que el sistema de dragalinas/apron feeder funcione adecuadamente este necesita de ciertas condiciones. Algunas de las unidades principales de la dragalina son: equipo de interfaz, casa de maquinaria, equipo de propulsión, base y planta de energía.

El equipo de la parte delantera generalmente se refiere al mástil, boom y el balde, así como a sus sistemas de cuerdas de soporte y funcionamiento. La casa de máquinas encierra toda la maquinaria de dragalina y los sistemas eléctricos. La casa también contiene un sistema de aire presurizado para eliminar el polvo o la acumulación de calor. El equipo de propulsión se utiliza para impulsar un mecanismo de leva y bastidor que produce una acción de marcha suave para la máquina. Una base integral tipo web lleva un conjunto de pivote central, cremallera y rodillo. Este equipo proporciona una estructura de distribución de carga para las obras superiores. La energía de Ca que se origina en una planta eléctrica externa se enruta a través de un cable de conexión a una subestación transformadora a bordo de la dragalina. Desde la subestación, la 
energía se dirige a los conjuntos $\mathrm{M}-\mathrm{G}$ y a la sala del transformador auxiliar. Los transformadores reducen el voltaje entrante para alimentar los equipos auxiliares, incluidos los compresores, las luces y los calentadores. Se realizaron tablas en Excel en las que se recopilaron datos históricos de la producción y costos de hace un año y los datos del 2017 hasta el mes de septiembre del libro de costos de la empresa.

En estas tablas se recopiló cada mes, día a día las horas operativas, el costo por turno de las dragalinas y sus equipos que conforman este sistema, con el fin de saber el número total de horas operativas de cada equipo, sus costos totales por turno del mes, costos totales por hora, y un factor hora por hora. El total de horas operativos se realizó mediante el cálculo del total de costos entre el costo por hora de cada equipo (dragalinas, apron feeder, backhoes, dozers, D11, camiones, etc.). Para las tablas de costos se realizó también un cálculo del factor hora por hora, el cual se realiza para mostrar el costo de una hora de los equipos que trabajan de la mano con las dragalinas. Se da a conocer un nuevo concepto nunca antes visto que es el factor de asignación de camiones, en los que la base de este es el ciclo de los camiones, el cual nos enfoca a analizar y ver qué tan influyente es el ciclo en la producción y costo de la operación, en este caso del sistema de dragalina/apron feeder, el cual será de gran ayuda para poder implementarlos en los demás sistemas (palas, load out, etc.) para poder tener una mejor asignación. Este nuevo concepto se diferencia del factor de camiones o factor carga que se implementa en la empresa, ya que el factor de carga lo hacen para dar una proyección de cuantos $\mathrm{BCM} / \mathrm{h}$ se pueden mover en un año por camión. Se realizó al final de las tablas de costos una tabla en la que se recopilaba el total de costos por turno (total shift cost), los BCM por turno (BCM/shift), el costo por BCM (\$/BCM) y el factor de asignación de camiones de cada día/turno generando graficas del costo por $\mathrm{BCM}(\$ / \mathrm{BCM})$ mensual del costo por BCM de cada equipo que conforma el sistema (dragalina, apron feeder, apron feeder transporter, apron feeder box, apron feeder E house, dozers, D11, camiones, back hoes, cargadores, etc.), en la que se hace una comparación con el Promedio de costos por BCM, mostrando que mes tuvo relevancia. Se generó un modelo de costo en el cual se lleva a analizar cuál es el punto óptimo para la asignación de camiones, teniendo en cuenta el número actual asignados y la afectación en los costos y producción del sistema de dragalinas/apron feeder, que 
se construyó basado en un resumen de las tablas del promedio costo por turno, llevando todo hacia las horas operativas esta vez no de la dragalina, sino de los apron feeder, creando un segundo factor de hora.

Construyendo a su vez una tabla mes por mes del factor de asignación de Camiones y desglosando cada uno de los tiempos que lo afecta promediando cada ítem para al final mostrarnos el total de BCM por turno (BCM/shift) y el costo por BCM (\$/BCM) que se genera en cada mes analizado. Tomando del libro amarillo o libro de costos de la empresa las horas de las dragalinas y apron Feeder las cuales fueron ajustadas para incluir en esta electricidad y ciertas labores del sistema.

A su vez, esta tabla nos describe el número de cargas, las horas operativas del sistema dragalina/apron feeder, las cargas por hora, altitud que se trabaja, los BCM del mes, BCM por hora, los camiones que se requieren y los que deben ser asignados para ser óptimos para nuestro sistema. Para luego arrojar una última tabla del modelo de costo la cual nos describe el número de camiones, mostrándonos el número óptimo de camiones, de costos y producción para la operación, mostrándonos lo influyente que es si le agregan más o menos camiones del optimo arrojado por el factor de asignación y describiendo equivalente en costos las horas operativas y el costo por turno de cada equipo del sistema de dragalinas/apron feeder en la mina.

Por último, se recrea el modelo en una curva de costos, en las que se muestra el impacto que genera tener un óptimo número de camiones a el costo y producción de esta, haciendo un comparativo con el número actual que se emplea en la empresa utilizando el factor de asignación de camiones, generando curvas de este modelo mes a mes para cada una de las dragalinas.

\section{Resultado}

En la mina se cuenta con un personal de primera, en el cual no solo le brindan calidad humana al practicante sino su sabiduría y experiencia en el campo de la minería, con el fin de obtener de la empresa su primera experiencia como profesional. El estar en constante relación con ingenieros extranjeros y nacionales con alta experiencia en el campo genera aptitudes como es hablar un lenguaje técnico y, a su vez, hablar una segunda lengua, en este caso el inglés, que hoy en día es una herramienta muy valiosa para el profesional, un hábito de compromiso, 
responsabilidad y sentido de pertenencia hacia la empresa, lo cual convierte al practicante en una persona capaz de enfrentarse a retos futuros, además de generar conciencia en mantener al medioambiente llevando de la mano una mejora continua para hacer de la minería cada día más viable y confiable. Gracias a esto y mucho más de parte de los ingenieros de planeación y todo su personal de la mina, se pudieron obtener resultados como: manejo básico del software AutoCAD, Carlson; experiencia en minería a cielo abierto; experiencia como ingeniera en el departamento de planeación; conocimiento adquirido sobre cómo funcionan las operaciones y procesos mineros en Drummond Ltd.; conocimiento básico en seguridad industrial; conocimiento básico en voladura y sus procesos; generalidades conceptuales y prácticas de topografía; relaciones interpersonales con el personal de la mina; aptitudes de responsabilidad, respeto y puntualidad; y afianzar conocimientos aprendidos durante la carrera como profesional.

Se realizó al final de las tablas de costos una tabla en la que se recopilaba el total de costos por turno (total shift cost), los BCM por turno (BCM/shift), el costo por $\mathrm{BCM}(\$ / \mathrm{BCM})$ y el factor de asignación de camiones de cada día/ turno, generando gráficas del costo por $\mathrm{BCM}(\$ / \mathrm{BCM})$ mensual, del costo por BCM de cada equipo que conforma el sistema (dragalina, apron feeder, apron feeder transporter, apron feeder box, apron feeder $E$ house, dozers, D11, camiones, backhoes, cargadores, etc,), en el que se hace una comparación con el promedio de costos por BCM, mostrando que mes tuvo relevancia.

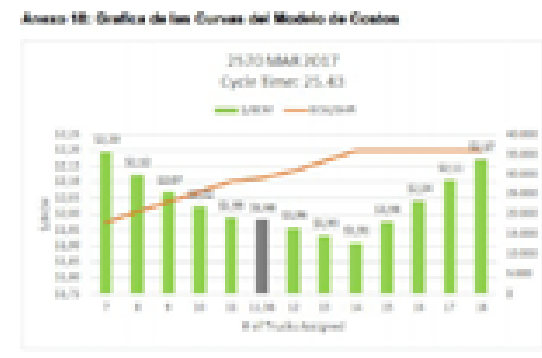

Figura 1. bcm explotados

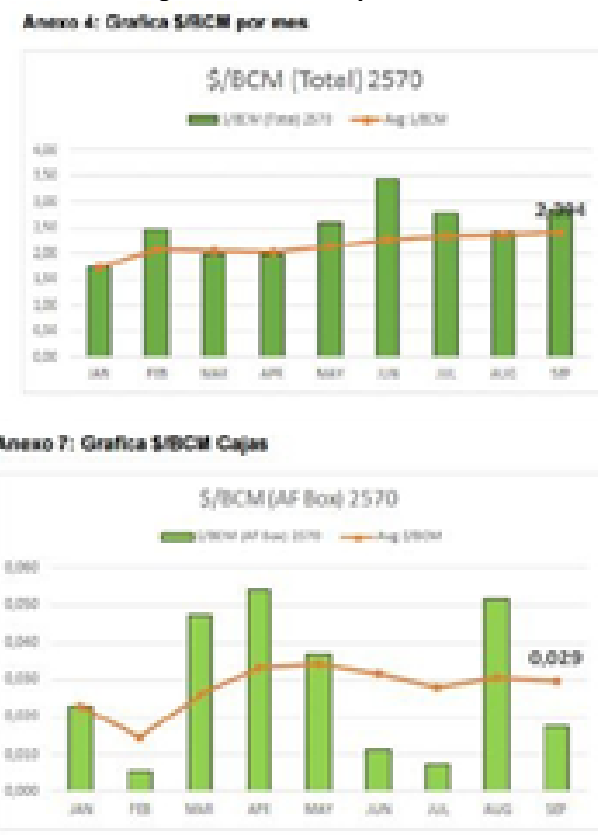

Figura 2. Curva de modelo de costos

Revista Agunkuyâa. Volumen 2: 69-75. 2017 ～ 73 
Modelo en una curva de costos, en la que se muestra el impacto que genera tener un óptimo número de camiones a costo y producción.

\section{Conclusiones}

Se conoció detalladamente el funcionamiento y operaciones de la mina. Se comprobó que la asignación de camiones en la operación de dragalinas/ apron feeder no es la más óptima, mostrando que afecta los costos y producción de esta. Se logró definir y mostrar el concepto del factor de asignación de camiones, mostrando que el factor ayuda para identificar cual es el número óptimo que debe tener la operación. Con este concepto se crearán nuevas bases para la empresa, para trabajar en la identificación de los ciclos y su mejora, la cual sería una gran ayuda para despacho, pues genera una asignación adecuada de los camiones, haciendo que la producción y costos de este sistema, y de todos en general en la mina, tenga un buen rendimiento a futuro. Se lograron identificar las características operativas del sistema dragalinas/apron feeder, las cuales siempre deben ser óptimas para que esta operación funcione adecuadamente, siendo la dragalina como equipo principal y equipo de apoyo (apron feeder, dozers, backhoes, D11, etc.) y los diseños implementados por el equipo de planeación y demás que se han de requerir para el buen funcionamiento de este sistema.

Se logró generar un modelo de costos, demostrando la saturación de la asignación de camiones que proporciona la tasa de producción óptima al menor costo por metro cúbico bancario, mostrando a su vez el impacto de los costos y producción que se genera con esta.

\section{Referencias}

Benton, K (2011). Tom. Dragline and Apron Feeder mining paper.May 17.

Corte Constitucional de Colombia (2014). Sentencia T849.

Rojas, Y. (2014). La historia de las áreas protegidas en Colombia, sus firmas de Gobierno y las alternativas para la gobernanza. Sociedad y Economía, 27, 155-176.

Ruiz, C. (2010). Alcance de la Investigación Cuantitativa. 